\title{
Spatial coherence of light emitted by thermalized ensembles of emitters coupled to surface waves
}

\author{
Elise Bailly $\odot$, Jean-Paul Hugonin, Benjamin Vest $\odot$, and Jean-Jacques Greffet $\odot^{*}$ \\ Université Paris-Saclay, Institut d'Optique Graduate School, CNRS, Laboratoire Charles Fabry, 91127 Palaiseau, France
}

(Received 9 August 2020; revised 5 July 2021; accepted 8 July 2021; published 13 August 2021)

\begin{abstract}
It has been discovered experimentally that light emitted by layers of photoexcited dye molecules or quantum dots deposited on metallic thin films is spatially coherent. Identifying the physical origin of this spatial coherence is a difficult task in the absence of a systematic procedure to model theoretically the field correlation function of the photoluminescence. The presence of strong coupling and the presence of delocalized plasmonic modes have been considered as possible candidates to explain the origin of the spatial coherence. Here, we use a general coherence-absorption relation recently derived to address this question.
\end{abstract}

DOI: 10.1103/PhysRevResearch.3.L032040

Light emission by ensembles of dye molecules or quantum dots has received a lot of attention recently. One of the driving forces of this interest is the design of light sources with improved properties in terms of spectral purity, energy efficiency. and versatility [1-14]. Another motivation is the investigation of the strong-coupling regime between light and matter [15-22]; phenomena have been reported such as the observation of photon Bose-Einstein condensation [23-28] or the discovery of the so-called cavity chemistry [6,17,21,22]. In this field, the existence of polaritons [29] plays a key role. An intriguing property of light emitted by a layer of photoexcited molecules on a silver thin film was discovered ten years ago [30]. It was shown that light produced by photoluminescence at two different points separated by several micrometers could produce interferences. This raises the question of the mechanism that produces a long-range correlation of the electromagnetic field at two points. To quantitatively assess the degree of spatial coherence, the natural tool is the spatial correlation of the electric fields. For a stationary random process, it can be cast in the form [31]

$$
\left\langle E_{j}\left(\mathbf{r}_{1}, \omega\right) E_{k}^{*}\left(\mathbf{r}_{2}, \omega^{\prime}\right)\right\rangle=W_{j k}\left(\mathbf{r}_{1}, \mathbf{r}_{2}, \omega\right) \delta\left(\omega-\omega^{\prime}\right),
$$

where $W_{j k}$ is the electric-field cross-spectral density tensor [32] and $E_{j}(\mathbf{r}, \omega)$ the time-domain Fourier transform of the electric field $E_{j}(\mathbf{r}, t)$, and $*$ denotes the complex conjugate. It is difficult to identify the physical origin of the long-range spatial correlation without an explicit model to compute this quantity. An analysis of the experimental data [30] suggested that the strong coupling plays a role in the onset of spatial coherence. Later studies [19] confirmed the existence of spatial coherence but it has been pointed out in Ref. [33] that strong coupling is not a necessary condition. Instead, it has

\footnotetext{
*Corresponding author: jean-jacques.greffet@institutoptique.fr

Published by the American Physical Society under the terms of the Creative Commons Attribution 4.0 International license. Further distribution of this work must maintain attribution to the author(s) and the published article's title, journal citation, and DOI.
}

been shown that the existence of surface waves appears to play a key role in building the spatial coherence. Furthermore, it has been observed that the visibility of the fringes depends on the pump intensity used in the photoluminescence process [28], an observation unexplained so far.

In this paper, we explore the origin of the spatial coherence in such a system by taking advantage of a recently-derived general coherence-absorption relation [34]. To address the issue of spatial coherence of light emitted by an ensemble of thermalized emitters, we consider the system studied in Ref. [30]. It consists of a thin layer of emitters on top of a metallic film. Our calculation will be performed using a layer of J-aggregated 5,5',6,6'-tetrachloro-1,1'-diethyl-3,3'di(4-sulfobutyl)-benzimidazolo-carbo cyanine (or TDBC). The emitters are deposited on a 45-nm silver layer on glass, as can be seen in Fig. 1. The dye layer is anisotropic and we use the dielectric permittivity model given in [30] to characterize it. Along the layer, the dielectric permittivity is written as

$$
\epsilon_{/ /}=\epsilon_{\mathrm{bg}}+A /\left(\omega_{0}^{2}-\left(\omega+i \Delta_{0}\right)^{2}\right),
$$

with $\omega_{0}=2.07 \mathrm{eV}, \epsilon_{\mathrm{bg}}=3.05, A=2 \mathrm{eV}^{2}$, and $\Delta_{0}=$ $14 \mathrm{meV}$. Perpendicular to the layer, $\epsilon_{\perp}=\epsilon_{\mathrm{bg}}$. The refractive index of the silver layer is described by a Drude model [30].

Thereafter, the cross-spectral density tensor is calculated in the framework of fluctuational electrodynamics [35]. A direct calculation was reported in the case of thermal emission [32]. By using the extension of the fluctuation-dissipation theorem to pumped materials in the thermalized regime [36,37], a similar approach is possible for photoluminescence. Here, we take an alternative approach and use a general coherence-absorption relation [34]. We consider two coherent monochromatic electric dipoles of amplitude unity located at $\mathbf{r}_{1}$ and $\mathbf{r}_{2}$ and polarized along the axis $j$ and $k$, respectively. We define the total absorbed power integrated over the TDBC layer when it is illuminated by these sources as $P_{j k}^{\mathrm{abs}}\left(\mathbf{r}_{1}, \mathbf{r}_{2}, \omega\right)$. If the sources were incoherent, this absorbed power would be equal to the sum of the absorptions generated by each source separately, $P_{j}^{\mathrm{abs}}\left(\mathbf{r}_{1}, \omega\right)$ and $P_{k}^{\mathrm{abs}}\left(\mathbf{r}_{2}, \omega\right)$. However, these two coherent sources located at $\mathbf{r}_{1}$ and $\mathbf{r}_{2}$ generate an 


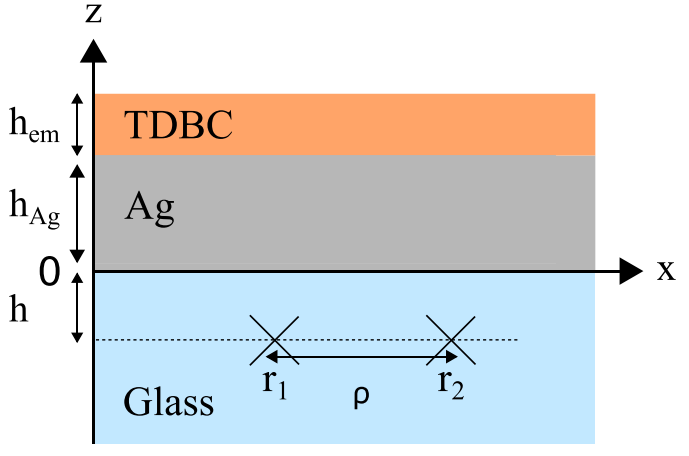

FIG. 1. Layout of the photoluminescence system. The structure consists in a layer of TDBC on top of a 45-nm-thick layer $\left(h_{\mathrm{Ag}}\right)$ of silver. These layers are deposited on a glass substrate. The crossspectral density tensor is calculated at the points $\mathbf{r}_{1}$ and $\mathbf{r}_{2}$, separated by the distance $\rho=\left|\mathbf{r}_{2}-\mathbf{r}_{1}\right|$ at a distance $h=1 \mu \mathrm{m}$ from the silver layer. The TDBC layer $\left(h_{\mathrm{em}}\right)$ is either 5-nm or 20-nm-thick.

interference pattern in the TDBC layer. As a consequence, the total absorbed power integrated over the TDBC layer is given by $[34,35]$

$$
\begin{aligned}
P_{j k}^{\mathrm{abs}}\left(\mathbf{r}_{1}, \mathbf{r}_{2}, \omega\right)= & P_{j}^{\mathrm{abs}}\left(\mathbf{r}_{1}, \omega\right)+P_{k}^{\mathrm{abs}}\left(\mathbf{r}_{2}, \omega\right) \\
& +2 \operatorname{Re}\left[P_{j k}^{\mathrm{mix}}\left(\mathbf{r}_{1}, \mathbf{r}_{2}, \omega\right)\right],
\end{aligned}
$$

where Re denotes the real part and $P_{j k}^{\operatorname{mix}}\left(\mathbf{r}_{1}, \mathbf{r}_{2}, \omega\right)$ is a complex term due to interferences called "mixed losses" [34,35]. The general coherence-absorption relation derived in [34] can then be written as

$$
W_{j k}\left(\mathbf{r}_{1}, \mathbf{r}_{2}, \omega\right)=4 \Theta\left(T_{\mathrm{q}}, \mu_{\mathrm{q}}\right) P_{j k}^{\operatorname{mix}}\left(\mathbf{r}_{1}, \mathbf{r}_{2}, \omega\right),
$$

with $\Theta\left(T_{\mathrm{q}}, \mu_{\mathrm{q}}\right)=\hbar \omega /\left[\exp \left(\left(\hbar \omega-\mu_{\mathrm{q}}\right) /\left(k_{\mathrm{B}} T_{\mathrm{q}}\right)\right)-1\right] \quad$ and $T_{\mathrm{q}}(\mathbf{r})$ and $\mu_{\mathrm{q}}(\mathbf{r})$ correspond to the temperature and photon chemical potential of the distribution of the excited electronic states of the TDBC molecules. If we consider that the two sources localized at $\mathbf{r}_{1}$ and $\mathbf{r}_{2}$ are polarized along the $\mathrm{x}$ axis, we can simplify relation (3) into

$$
P_{x x}^{\mathrm{abs}}\left(\mathbf{r}_{1}, \mathbf{r}_{2}, \omega\right)=2\left(P_{x}^{\mathrm{abs}}\left(\mathbf{r}_{0}, \omega\right)+P_{x x}^{\mathrm{mix}}\left(\mathbf{r}_{1}, \mathbf{r}_{2}, \omega\right)\right),
$$

with $\mathbf{r}_{0}=\left(\mathbf{r}_{1}+\mathbf{r}_{2}\right) / 2$. The system being invariant along the $\mathrm{x}$ and y axis, $P_{x}^{\text {abs }}\left(\mathbf{r}_{0}, \omega\right)=P_{x}^{\text {abs }}\left(\mathbf{r}_{1}, \omega\right)=P_{x}^{\text {abs }}\left(\mathbf{r}_{2}, \omega\right)$. Furthermore, $P_{x x}^{\operatorname{mix}}$ is real when $z_{1}=z_{2}$ [34]. From (4) and (5), we derive the following relation:

$$
\frac{W_{x x}\left(\mathbf{r}_{1}, \mathbf{r}_{2}, \omega\right)}{W_{x x}\left(\mathbf{r}_{0}, \mathbf{r}_{0}, \omega\right)}=\frac{P_{x x}^{\text {abs }}\left(\mathbf{r}_{1}, \mathbf{r}_{2}, \omega\right)}{2 P_{x}^{\text {abs }}\left(\mathbf{r}_{0}, \omega\right)}-1,
$$

Both the absorbed power and the cross-spectral density tensor are normalized by their values at $\mathbf{r}_{0}=\left(\mathbf{r}_{1}+\mathbf{r}_{2}\right) / 2$. Equation (6) establishes an equivalence between a correlation function of fluctuating fields and absorption of deterministic coherent fields. It provides a means to obtain experimentally the spatial correlation of the fields by measuring an absorption. It also provides a tool that has been missing so far to analyze theoretically the spatial coherence of the fields emitted by thermalized sources.

As the spatial coherence is linked to the extension and number of modes excited by the source, we first study the modes of the system by plotting the reflectivity (Fig. 2) when

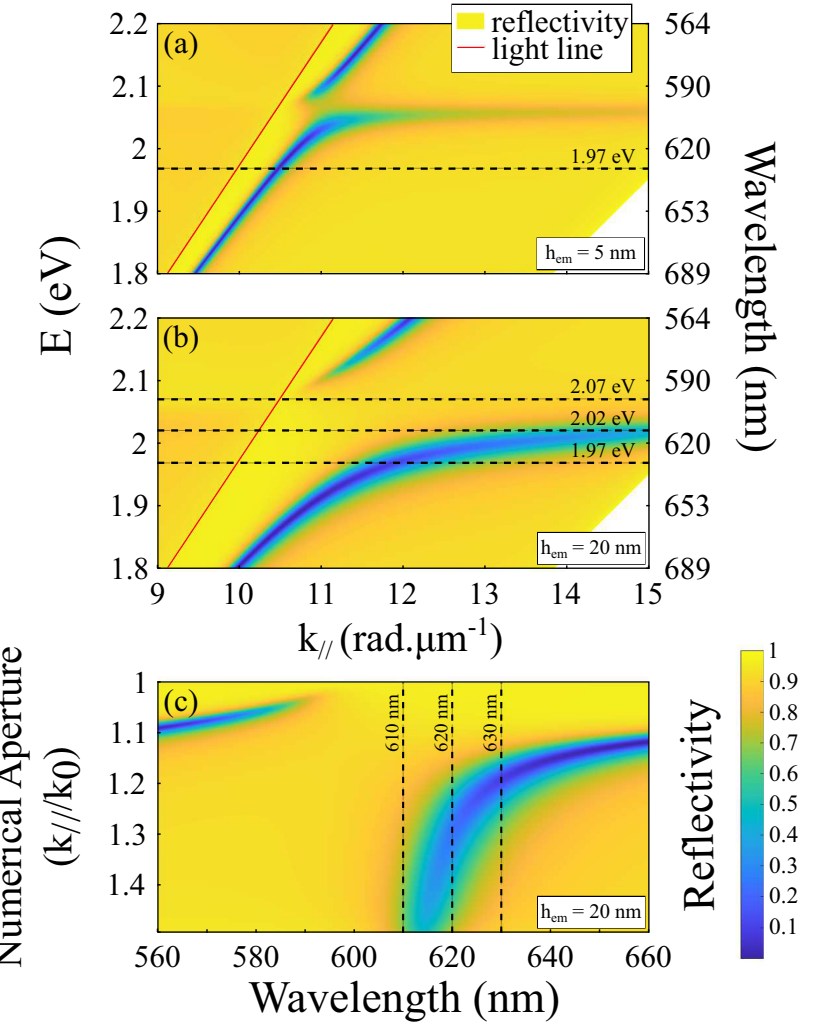

FIG. 2. Reflectivity maps of the structure. [(a),(b)] As a function of the energy and the in-plane wave vector for (a) $h_{\mathrm{em}}=5 \mathrm{~nm}$ and (b) $h_{\mathrm{em}}=20 \mathrm{~nm}$. (c) As a function of the numerical aperture (defined as $k_{/ /} / k_{0}$, with $\left.k_{0}=2 \pi / \lambda\right)$ and the wavelength for $h_{\mathrm{em}}=20 \mathrm{~nm}$. This second graph is another graphical representation of the gap region between $1.88 \mathrm{eV}$ and $2.21 \mathrm{eV}$.

the system is excited by a plane wave propagating from the bottom in the glass substrate towards the metal layer in a Kretschmann configuration. It is seen that the reflectivity has a dip for wave vectors slightly larger than $\omega / c$. This is a signature of the resonant excitation of the surface plasmon propagating at the interface silver/TDBC/air [38]. The second feature clearly observed is the anticrossing between the plasmon dispersion relation and the exciton, opening a gap between $2.03 \mathrm{eV}$ and $2.09 \mathrm{eV}$ in Fig. 2 (a) $\left(h_{\mathrm{em}}=5 \mathrm{~nm}\right)$ and between $1.93 \mathrm{eV}$ and $2.11 \mathrm{eV}$ in Fig. 2(b) $\left(h_{\mathrm{em}}=20 \mathrm{~nm}\right)$. The gap opening is a signature of the strong coupling and the formation of exciton-polaritons. We play on the coupling by varying the thickness of the TDBC layer [Figs. 2(a) and 2(b)]. It is seen on the dispersion relation that the modes at $1.97 \mathrm{eV}$ appear to have a plasmonic (weak coupling with TDBC) character in Fig. 2(a) and a polaritonic (strong coupling with TDBC molecules) character in Fig. 2(b). Figure 2(c) reproduces the reflectivity of the same system, for $h_{\mathrm{em}}=20 \mathrm{~nm}$; it is plotted as a function of wavelength and numerical aperture in order to ease the comparison with the original article [30].

We now calculate the normalized cross-spectral density tensor using Eq. (6) at different frequencies to find out how the structure of the modes affects the spatial coherence. The calculations have been obtained by placing two sources at two points $\mathbf{r}_{1}$ and $\mathbf{r}_{2}$ (both along the $\mathrm{x}$ axis) in the glass, at a distance $h=1 \mu \mathrm{m}$ from the silver layer. To mimic the 


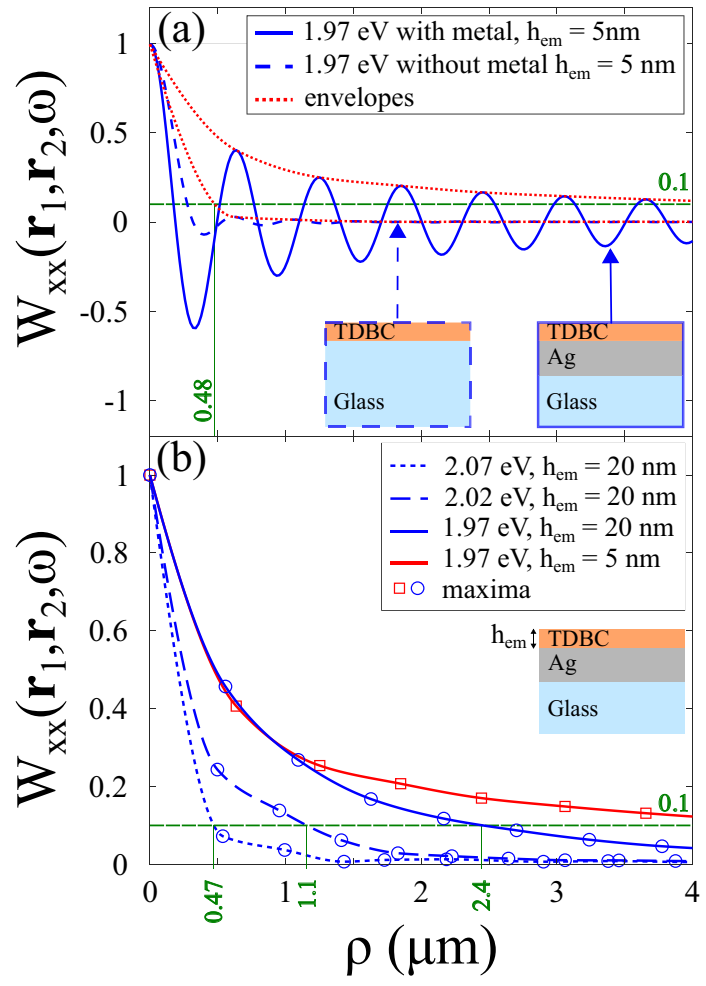

FIG. 3. Diagonal elements of the electric-field cross-spectral density tensor $W_{x x}\left(\mathbf{r}_{1}, \mathbf{r}_{2}, \omega\right)$ as a function of the distance $\rho$ between positions $\mathbf{r}_{1}$ and $\mathbf{r}_{2}$. (a) At $1.97 \mathrm{eV}$, with and without the silver layer and $h_{\mathrm{em}}=5 \mathrm{~nm}$. (b) Envelopes only, at $1.97 \mathrm{eV}$ with $h_{\mathrm{em}}=5 \mathrm{~nm}$ and $20 \mathrm{~nm}$, and at $2.02 \mathrm{eV}$ and $2.07 \mathrm{eV}$ with $h_{\mathrm{em}}=20 \mathrm{~nm}$ and with the silver layer. All plots are normalized by their maximum value at $\rho=0$.

experiment of [30], the sources have been placed at that distance to filter out the evanescent waves with wave vector much larger than $n_{\text {glass }} \omega / c$. In doing so, the surface plasmon polariton at the interface glass/metal does not intervene in the correlation function (see [38]). The absorption is computed by means of the difference of the Poynting fluxes at the interfaces Air/TDBC and TDBC/Ag. The envelopes of the oscillating correlation functions have been plotted as a function of $\rho=$ $\left|\mathbf{r}_{2}-\mathbf{r}_{1}\right|$ in Figs. 3(a) (red dotted lines) and 3(b) and they will be denoted as $\mathcal{E}\left(\left|W_{x x}\left(\mathbf{r}_{1}, \mathbf{r}_{2}, \omega\right)\right|\right)$ in the following. They will be used to quantify the correlation lengths in the different cases with $\rho_{0.1}$ the value of $\rho$ for which $\mathcal{E}\left(\left|W_{x x}\left(\mathbf{r}_{1}, \mathbf{r}_{2}, \omega\right)\right|\right)=$ 0.1 . The larger $\rho_{0.1}$, the larger the correlation length. All the results for the different cases studied are gathered in Table I.

We first analyze the role of the silver layer. In Fig. 3(a), we compare the cross-spectral density $W_{x x}\left(\mathbf{r}_{1}, \mathbf{r}_{2}, \omega\right)$ at $1.97 \mathrm{eV}$ (i.e., $630 \mathrm{~nm}$ ) with and without a silver layer. It is seen that the correlation length is much larger in the presence of the layer $\left(\rho_{0.1}=5.0 \mu \mathrm{m}\right)$ than without $\left(\rho_{0.1}=0.48 \mu \mathrm{m}\right)$. At this frequency, the reflectivity exhibits a surface-plasmon resonance [Fig. 2(a)], suggesting that the presence of a surface mode plays a key role. Note also that these plots are obtained in the weak coupling zone. This shows unambiguously that it is not necessary to be in the strong-coupling regime to observe spatial coherence.

We can verify the role of the surface wave by calculating the correlation at $2.07 \mathrm{eV}$, in the gap region [Figs. 2(b) and $3(\mathrm{~b}), h_{\mathrm{em}}=20 \mathrm{~nm}$ ], where no surface mode can be excited (note however that if the permittivity is isotropic, an additional polaritonic branch appears in the gap; see [38]). Despite the presence of the silver layer, $\mathcal{E}\left(\left|W_{x x}\left(\mathbf{r}_{1}, \mathbf{r}_{2}, \omega_{2.07 \mathrm{eV}}\right)\right|\right)<0.1$ for $\rho \geqslant 0.47 \mu \mathrm{m}$. The comparison between the curves at $1.97 \mathrm{eV}$ and at $2.07 \mathrm{eV}$ confirms that exciting surface waves increases the range of spatial coherence.

We now plot $W_{x x}\left(\mathbf{r}_{1}, \mathbf{r}_{2}, \omega\right)$ at $2.02 \mathrm{eV}$ (for $h_{\mathrm{em}}=20 \mathrm{~nm}$ ), an energy where the reflectivity map shows that the minima plot reaches an asymptote due to the strong coupling between the plasmon and the exciton. It is seen in Fig. 3(b) that the correlation decays very fast $\left[\mathcal{E}\left(\left|W_{x x}\left(\mathbf{r}_{1}, \mathbf{r}_{2}, \omega_{2.02 \mathrm{eV}}\right)\right|\right)<0.1\right.$ for $\rho \geqslant 1.1 \mu \mathrm{m}]$. Here, the origin of the low-coherence length is not the lack of surface modes but a large number of excited modes in the interval $\Delta k_{x}$. A dipole source emitting at this frequency can excite many modes with different wave vectors $k_{x}$ in an interval $\Delta k_{x}$. These different modes dephase on a length scale on the order of $1 / \Delta k_{x}$. This is in marked contrast with the fewer surface modes excited at $1.97 \mathrm{eV}$ in the weakcoupling regime [Figs. 2(a) and 3(a)].

If we compare the reflectivities at $1.97 \mathrm{eV}$ and at $2.02 \mathrm{eV}$ the main difference lies in the widths $\Delta k$ of the reflectivity dip. $\Delta k_{1.97 \mathrm{eV}} \ll \Delta k_{2.02 \mathrm{eV}}$ leads to a higher spatial coherence at $1.97 \mathrm{eV}$ than at $2.02 \mathrm{eV}$, which is confirmed by Fig. 3(b). Note that at $1.97 \mathrm{eV}, \Delta k$ is thinner in the weak coupling regime $\left(h_{\mathrm{em}}=5 \mathrm{~nm}\right)$ compared to the strong coupling regime $\left(h_{\mathrm{em}}=20 \mathrm{~nm}\right)$. We thus obtain that $\rho_{0.1(1.97 \mathrm{eV}-5 \mathrm{~nm})}>$ $\rho_{0.1(1.97 \mathrm{eV}-20 \mathrm{~nm}) \text {. }}$

In summary, we have shown the crucial role of surface waves in the spatial coherence. We also note that the strongcoupling regime is not a necessary condition to observe spatial coherence in the emitted fields, as was also reported in more recent experiments $[19,33]$.

To identify the mechanism responsible for the buildup of spatial coherence from a mere superposition of uncorrelated spontaneous emission events, we go back to the basics of light emission by thermalized systems in the framework of fluctuational electrodynamics. Fluctuating current densities in a given volume element excite the electromagnetic modes. Some of the modes are leaky surface modes, which can prop-

TABLE I. $\rho_{0.1}$, i.e., values of $\rho$ for which the envelopes of the correlation $\mathcal{E}\left(\left|W_{x x}\left(\mathbf{r}_{1}, \mathbf{r}_{2}, \omega\right)\right|\right.$ are equal to 0.1 , for three different energies $(1.97 \mathrm{eV}, 2.02 \mathrm{eV}$, and $2.07 \mathrm{eV})$ when the dye layer is deposited on silver ("with metal") or glass ("without metal").

\begin{tabular}{lcccc}
\hline \hline Energy $(\mathrm{eV})$ & 1.97 & 1.97 & 2.02 & 2.07 \\
\hline$h_{\mathrm{em}}(\mathrm{nm})$ & 5 & 20 & 20 & 20 \\
$\rho_{0.1}(\mu \mathrm{m})$, with metal & 5.0 & 2.4 & 1.1 & 0.47 \\
$\rho_{0.1}(\mu \mathrm{m})$, without metal & 0.48 & & \\
\hline \hline
\end{tabular}




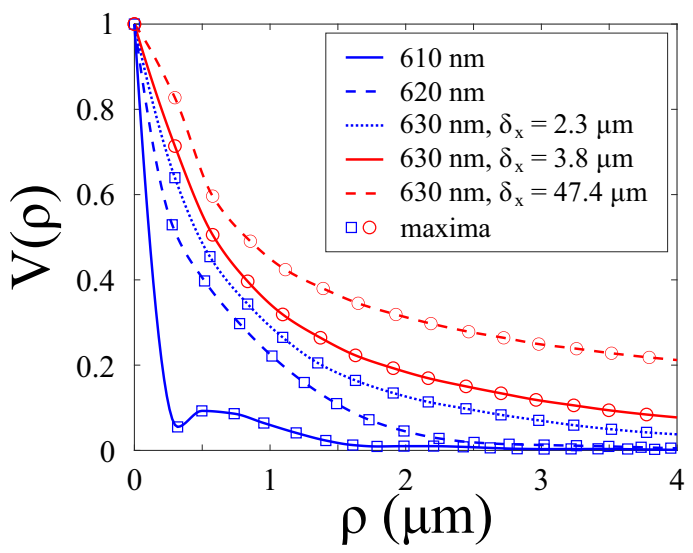

FIG. 4. Representation of the envelopes of $V(\rho)=$ $\left|W_{x x}\left(\mathbf{r}_{1}, \mathbf{r}_{2}, \omega\right) / W_{x x}\left(\mathbf{r}_{0}, \mathbf{r}_{0}, \omega\right)\right|$ as a function of the distance $\rho$. $V(\rho)$ can be related to fringes visibility in Young's slits type interference experiments, slits being separated by distance $\rho$. Squares and circles are local maxima of $V(\rho)$ and lines are interpolations of those maxima and guides for the eye. Envelopes are plotted (i) for three different wavelengths (blue squares and lines) at 610,620 , and $630 \mathrm{~nm}$; (ii) at $630 \mathrm{~nm}$ for other values of the decay length of the plasmon (red circles and lines), corresponding to either bleaching $\left(\delta_{x}=3.8 \mu \mathrm{m}\right)$ or gain in the dye layer $\left(\delta_{x}=47.4 \mu \mathrm{m}\right)$. The TDBC layer is 20 -nm-thick.

agate along the surface and radiate into the substrate. These modes contribute to the electric field at two different points along the interface generating a correlation of the field at these two points. It is the same mechanism that produces spatial coherence of thermal fields in the presence of surface waves [32,39]. When for a given frequency, several modes corresponding to several wave vectors are simultaneously excited, as it happens when the dispersion relation flattens, the different modes dephase over propagation. Finally, we note that there are no leaky surface modes in the gap.

We now compare the calculations of the cross-spectral density reported in this paper to previously reported experimental data [30]. The system is the same as the one depicted in Fig. 1. The spatial correlation at positions $\mathbf{r}_{1}$ and $\mathbf{r}_{2}$ was experimentally characterized by measuring the visibility of interference fringes formed with the fields diffracted by two slits. The fringe visibility can be modelled by the modulus of the normalized cross-spectral density tensor [40,41] that we call $V(\rho)$ where $\rho$ is the distance between the two slits. It is an oscillating function and we report the loci of its maxima in Fig. 4 as a function of $\rho$ for three different wavelengths.
$V(\rho)$ is higher at $630 \mathrm{~nm}$ (blue dotted line) than at $610 \mathrm{~nm}$ (blue solid line), which is consistent with the experimental data; $610 \mathrm{~nm}$ corresponds to $2.03 \mathrm{eV}$, an energy close to the gap where $\Delta k$ increases [Fig. 2(c)].

It is noteworthy that the values of visibility that we obtained numerically are smaller than the experimental values. Firstly, we attribute this difference to the fact that this model does not account for the imaging system nor for the presence of a spatial frequency filter. Secondly, we did not take into account the pumping of the dyes in the dielectric permittivity model given in [30]. It has indeed been observed that increasing pump fluence leads to a gradual build-up of the degree of spatial coherence [20]. To explain this observation, we first note that when increasing the pumping, the losses in the dye layer are progressively reduced until they are transformed into gain, which leads to an increase of the decay length $\delta_{x}$ (defined as $1 / k^{\prime \prime}$ ) of the plasmon and thus to an increase of the correlation length (see [38]). Even though the precise experimental value of the losses in the dye layer is unknown, it is possible to study this behavior with the reduction of the losses by changing the value of $\Delta_{0}$ in (2). The interpolation of the maxima of the modulus of $W_{x x}\left(\mathbf{r}_{1}, \mathbf{r}_{2}, \omega\right), V(\rho)$, at $630 \mathrm{~nm}$ is shown in Fig. 4 without losses in the dye layer (red solid line) and with gain (red dotted line). For $\rho=4 \mu \mathrm{m}$, the visibility is multiplied by at least a factor 2 without losses in the dye layer and by a factor 5.6 with gain.

In conclusion, a general coherence-absorption relation has been used to compute the cross-spectral density of the field emitted by an ensemble of thermalized molecules above a metallic-thin silver film. We find that the origin of the spatial coherence of the electromagnetic field can be attributed to the presence of leaky surface plasmons propagating along the interface. We stress that the spatial coherence can be explained without invoking localized states as in [30]. A further discussion of the arguments put forward in Ref. [30] is given within the Supplemental Material [38]. We also find that the spatial coherence length is larger when the optical pumping increases the decay length of the surface plasmons. When the system enters the strong coupling regime, the electromagnetic field has a reduced spatial coherence. In the gap, it is due to the absence of surface waves; close to the band edge, it is due to the large number of excited exciton polaritons with different wave vectors.

This work is supported by the French National Agency (ANR) (ANR-17-CE24-0046). J.-J.G. acknowledges the support of Institut Universitaire de France (IUF).
[1] W. L. Barnes, Electromagnetic crystals for surface plasmon polaritons and the extraction of light from emissive devices, J. Lightwave Technol. 17, 2170 (1999).

[2] R. Paiella, Tunable surface plasmons in coupled metallodielectric multiple layers for light-emission efficiency enhancement, Appl. Phys. Lett. 87, 111104 (2005).

[3] J. B. Khurgin, G. Sun, and R. A. Soref, Enhancement of luminescence efficiency using surface plasmon polaritons: Figures of merit, J. Opt. Soc. Am. B 24, 1968 (2007).
[4] G. Sun, J. B. Khurgin, and R. A. Soref, Plasmonic lightemission enhancement with isolated metal nanoparticles and their coupled arrays, J. Opt. Soc. Am. B 25, 1748 (2008).

[5] G. Vecchi, V. Giannini, and J. Gómez Rivas, Shaping the Fluorescent Emission by Lattice Resonances in Plasmonic Crystals of Nanoantennas, Phys. Rev. Lett. 102, 146807 (2009).

[6] H. Aouani, O. Mahboub, E. Devaux, H. Rigneault, T. W. Ebbesen, and J. Wenger, Plasmonic antennas for directional sorting of fluorescence emission, Nano Lett. 11, 2400 (2011). 
[7] S. R. K. Rodriguez, G. Lozano, M. A. Verschuuren, R. Gómez, K. Lambert, B. De Geyter, A. Hassinen, D. Van Thourhout, Z. Hens, and J. Gómez Rivas, Quantum rod emission coupled to plasmonic lattice resonances: A collective directional source of polarized light, Appl. Phys. Lett. 100, 111103 (2012).

[8] K. Guo, G. Lozano, M. A. Verschuuren, and J. Gómez Rivas, Control of the external photoluminescent quantum yield of emitters coupled to nanoantenna phased arrays, J. Appl. Phys. 118, 073103 (2015)

[9] I. Staude, V. V. Khardikov, N. T. Fofang, S. Liu, M. Decker, D. N. Neshev, T. S. Luk, I. Brener, and Y. S. Kivshar, Shaping photoluminescence spectra with magnetoelectric resonances in all-dielectric nanoparticles, ACS Photonics 2, 172 (2015).

[10] G. Lozano, S. R. K. Rodriguez, M. A. Verschuuren, and J. Gómez Rivas, Metallic nanostructures for efficient LED lighting, Light Sci. Appl. 5, e16080 (2016).

[11] J. Bohn, T. Bucher, K. E. Chong, A. Komar, D.-Y. Choi, D. N. Neshev, Y. S. Kivshar, T. Pertsch, and I. Staude, Active tuning of spontaneous emission by Mie-resonant dielectric metasurfaces, Nano Lett. 18, 3461 (2018).

[12] S. Liu, A. Vaskin, S. Addamane, B. Leung, M.-C. Tsai, Y. Yang, P. P. Vabishchevich, G. A. Keeler, G. Wang, X. He et al., Light-emitting metasurfaces: Simultaneous control of spontaneous emission and far-field radiation, Nano Lett. 18, 6906 (2018).

[13] J. Liu, W. Wang, D. Wang, J. Hu, W. Ding, R. D. Schaller, G. C. Schatz, and T. W. Odom, Spatially defined molecular emitters coupled to plasmonic nanoparticle arrays, Proc. Natl. Acad. Sci. U.S.A. 116, 5925 (2019).

[14] A. Vaskin, R. Kolkowski, A. F. Koenderink, and I. Staude, Light-emitting metasurfaces, Nanophotonics 8, 1151 (2019).

[15] C. Weisbuch, M. Nishioka, A. Ishikawa, and Y. Arakawa, Observation of the Coupled Exciton-Photon Mode Splitting in a Semiconductor Quantum Microcavity, Phys. Rev. Lett. 69, 3314 (1992).

[16] J. Bellessa, C. Bonnand, J. C. Plenet, and J. Mugnier, Strong Coupling between Surface Plasmons and Excitons in an Organic Semiconductor, Phys. Rev. Lett. 93, 036404 (2004).

[17] J. Dintinger, S. Klein, F. Bustos, W. L. Barnes, and T. W. Ebbesen, Strong coupling between surface plasmon-polaritons and organic molecules in subwavelength hole arrays, Phys. Rev. B 71, 035424 (2005).

[18] D. E. Gómez, K. C. Vernon, P. Mulvaney, and T. J. Davis, Surface plasmon mediated strong exciton-photon coupling in semiconductor nanocrystals, Nano Lett. 10, 274 (2010).

[19] L. Shi, T. K. Hakala, H. T. Rekola, J.-P. Martikainen, R. J. Moerland, and P. Törmä, Spatial Coherence Properties of Organic Molecules Coupled to Plasmonic Surface Lattice Resonances in the Weak and Strong Coupling Regimes, Phys. Rev. Lett. 112, 153002 (2014).

[20] P. Törmä and W. L. Barnes, Strong coupling between surface plasmon polaritons and emitters: A review, Rep. Prog. Phys. 78, 13901 (2015).

[21] J. George, S. Wang, T. Chervy, A. Canaguier-Durand, G. Schaeffer, J.-M. Lehn, J. A. Hutchison, C. Genet, and T. W. Ebbesen, Ultra-strong coupling of molecular materials: spectroscopy and dynamics, Faraday Discuss. 178, 281 (2015).

[22] J. George, T. Chervy, A. Shalabney, E. Devaux, H. Hiura, C. Genet, and T. W. Ebbesen, Multiple Rabi Splittings under
Ultrastrong Vibrational Coupling, Phys. Rev. Lett. 117, 153601 (2016).

[23] S. R. K. Rodriguez, J. Feist, M. A. Verschuuren, F. J. Garcia Vidal, and J. Gómez Rivas, Thermalization and Cooling of Plasmon-Exciton Polaritons: Towards Quantum Condensation, Phys. Rev. Lett. 111, 166802 (2013).

[24] J. Klaers, F. Vewinger, and M. Weitz, Thermalization of a twodimensional photonic gas in a 'white wall' photon box, Nat. Phys. 6, 512 (2010).

[25] J. Klaers, J. Schmitt, F. Vewinger, and M. Weitz, Bose-Einstein condensation of photons in an optical microcavity, Nature (London) 468, 545 (2010).

[26] K. S. Daskalakis, S. A. Maier, R. Murray, and S. Kéna-Cohen, Nonlinear interactions in an organic polariton condensate, Nat. Mater. 13, 271 (2014).

[27] J.-P. Martikainen, M. O. J. Heikkinen, and P. Törmä, Condensation phenomena in plasmonics, Phys. Rev. A 90, 053604 (2014).

[28] T. K. Hakala, A. J. Moilanen, A. I. Väkeväinen, R. Guo, J.-P. Martikainen, K. S. Daskalakis, H. T. Rekola, A. Julku, and P. Törmä, Bose-Einstein condensation in a plasmonic lattice, Nat. Phys. 14, 739 (2018).

[29] J. J. Hopfield, Theory of the contribution of excitons to the complex dielectric constant of crystals, Phys. Rev. 112, 1555 (1958).

[30] S. Aberra Guebrou, C. Symonds, E. Homeyer, J. C. Plenet, Y. N. Gartstein, V. M. Agranovich, and J. Bellessa, Coherent Emission from a Disordered Organic Semiconductor Induced by Strong Coupling with Surface Plasmons, Phys. Rev. Lett. 108, 066401 (2012).

[31] L. Landau and E. Lifshitz, Statistical Physics, Vol. 5 (Elsevier Science, New York, 2013).

[32] R. Carminati and J. J. Greffet, Near-Field Effects in Spatial Coherence of Thermal Sources, Phys. Rev. Lett. 82, 1660 (1999).

[33] L. Shi, X. Yuan, Y. Zhang, T. Hakala, S. Yin, D. Han, X. Zhu, B. Zhang, X. Liu, P. Törmä, W. Lu, and J. Zi, Coherent fluorescence emission by using hybrid photonic-plasmonic crystals, Laser Photonics Rev. 8, 717 (2014).

[34] D. Tihon, S. Withington, E. Bailly, B. Vest, and J.-J. Greffet, General relation between spatial coherence and absorption, Opt. Express 29, 425 (2021).

[35] S. Rytov, Y. Kravtsov, and V. I. Tatarskii, Priniciples of Statistical Radiophysics. 3. Elements of Random Fields (Springer, Berlin, 1989).

[36] C. H. Henry and R. F. Kazarinov, Quantum noise in photonics, Rev. Mod. Phys. 68, 801 (1996).

[37] J.-J. Greffet, P. Bouchon, G. Brucoli, and F. Marquier, Light Emission by Nonequilibrium Bodies: Local Kirchhoff Law, Phys. Rev. X 8, 21008 (2018).

[38] See Supplemental Material at http://link.aps.org/supplemental/ 10.1103/PhysRevResearch.3.L032040 for a description of the modes structure and its influence on the coherence, the influence of pumping on the coherence length and a brief discussion of the interferences observed with a localized illumination between the Young's slits.

[39] J.-J. Greffet, R. Carminati, K. Joulain, J.-P. Mulet, S. Mainguy, and Y. Chen, Coherent emission of light by thermal sources, Nature (London) 416, 61 (2002).

[40] L. Mandel and E. Wolf, Spectral coherence and the concept of cross-spectral purity, J. Opt. Soc. Am. 66, 529 (1976).

[41] J. Goodman, Statistical Optics (Wiley, Hoboken, NJ, 2015). 